\title{
A note on the calibration of pressure-velocity sound intensity probes
}

\author{
Jacobsen, Finn; Jaud, Virginie
}

Published in:

Acoustical Society of America. Journal

Link to article, DOI:

10.1121/1.2214144

Publication date:

2006

Document Version

Publisher's PDF, also known as Version of record

Link back to DTU Orbit

Citation (APA):

Jacobsen, F., \& Jaud, V. (2006). A note on the calibration of pressure-velocity sound intensity probes. Acoustical Society of America. Journal, 120(2), 830-837. https://doi.org/10.1121/1.2214144

\section{General rights}

Copyright and moral rights for the publications made accessible in the public portal are retained by the authors and/or other copyright owners and it is a condition of accessing publications that users recognise and abide by the legal requirements associated with these rights.

- Users may download and print one copy of any publication from the public portal for the purpose of private study or research.

- You may not further distribute the material or use it for any profit-making activity or commercial gain

- You may freely distribute the URL identifying the publication in the public portal

If you believe that this document breaches copyright please contact us providing details, and we will remove access to the work immediately and investigate your claim. 


\title{
A note on the calibration of pressure-velocity sound intensity probes ${ }^{\text {a) }}$
}

\author{
Finn Jacobsen ${ }^{\text {b) }}$ and Virginie Jaud ${ }^{\text {c) }}$ \\ Acoustic Technology, Ørsted-DTU, Technical University of Denmark, Building 352, Ørsteds Plads, \\ DK-2800 Kgs. Lyngby, Denmark
}

(Received 20 January 2006; revised 18 May 2006; accepted 20 May 2006)

\begin{abstract}
A pressure-velocity sound intensity probe is a device that combines a pressure microphone with a particle velocity transducer. Various methods of calibrating such sound intensity probes are examined: a far field method that requires an anechoic room, a near field method that involves sound emitted from a small hole in a plane baffle, a near field method where the sound is emitted from a hole in a spherical baffle, and a method that involves an impedance tube. The performance of the two near field methods is examined both in an anechoic room and in various ordinary rooms. It is shown that whereas reflections from the edges from a plane baffle disturb the calibration, the method based on a spherical baffle gives acceptable results in a wide frequency range even when the calibration is carried out in a small office, provided that the distance between the hole and the device under test is about $5 \mathrm{~cm}$. (C) 2006 Acoustical Society of America. [DOI: 10.1121/1.2214144]
\end{abstract}

PACS number(s): 43.58.Vb, 43.58.Fm, 43.20.Ye [AJZ]

Pages: $830-837$

\section{INTRODUCTION}

Until recently direct measurement of the acoustic particle velocity in air was almost impossible. However, a pressure-velocity (" $p-u$ ") sound intensity probe based on a particle velocity transducer called the "Microflown" combined with a small pressure microphone has now been available for some years, ${ }^{1,2}$ and recent results seem to indicate that it is viable. ${ }^{3}$ The potential applications of such a device include the applications of the conventional, standardized sound intensity measurement technique based on pairs of matched condenser microphones (the " $p-p$ method"), ${ }^{4,5}$ that is, measurement of sound power, identification and ranking of sources, visualization of sound fields, measurement of transmission loss, identification of transmission paths, etc. ${ }^{6}$ However, there seem to be additional potential applications, for instance measurement of sound absorption, ${ }^{7,8}$ and near field acoustic holography ${ }^{9}$ and other inverse source identification techniques. ${ }^{10,11}$ It is also potentially useful that a particle velocity transducer placed close to a vibrating surface is less affected by background noise than a pressure microphone. ${ }^{12,13}$ Most of these applications rely on accurate calibration of the two transducers of the $p-u$ intensity probe, and for some applications the phase calibration has been shown to be of critical importance. ${ }^{3,8}$ However, whereas calibration of the pressure microphones of a $p-p$ sound intensity probe is fairly simple and unproblematic, ${ }^{4-6}$ there is no established method of calibrating a $p-u$ probe. ${ }^{6}$ The two trans-

\footnotetext{
${ }^{a)}$ Portions of this work were presented in "Measurement of sound intensity: $p-u$ probes versus $p-p$ probes," Proceedings of Noise and Vibration Emerging Methods 2005, Saint Raphaël, France, April 2005, and in "Calibration of $p-u$ intensity probes," Proceedings of Euronoise 2006, Tampere, Finland, May 2006.

${ }^{b)}$ Author to whom correspondence should be addressed; Electronic mail: fja@oersted.dtu.dk

${ }^{c)}$ Electronic mail: virginie.jaud@gmail.com
}

ducers are completely different and cannot be expected to have the same amplitude and phase response, and therefore it is necessary to determine a correction of one of them relative to the other. Since condenser microphones are well behaved and easy to calibrate with a reference microphone the obvious choice is to calibrate the particle velocity transducer relative to the pressure transducer of the $p$ - $u$ probe. ${ }^{6}$

Calibration of a $p-u$ intensity probe involves exposing it to a sound field with a known relationship between the sound pressure and the particle velocity. A number of methods have been described in the literature. In the underwater acoustics community, where the $p-u$ intensity measurement principle is more established than in air-borne sound, a common calibration technique involves the use of a vertical water-filled tube in which the water-air interface provides an almost perfect pressure-release termination and thus a known relation between the pressure and the velocity in the sound field in the tube. ${ }^{14-16}$ A similar method can be used in air with a rigidly terminated tube, ${ }^{1,2}$ but since modes of higher order must be avoided the frequency range is limited to a few kilohertz. One can also calibrate in a large anechoic room. ${ }^{3}$ However, there is obviously a need for a calibration technique that covers a substantial part of the audible frequency range and can be used in the field. One possible such field calibration method involves measuring relatively near a small loudspeaker in an ordinary room and removing the influence of room reflections using a time-selective technique. ${ }^{7}$ However, because of the resulting truncation of the impulse response this method is not accurate at low frequencies. ${ }^{7}$

If the measurement takes place very close to a source then reflections from the surroundings can perhaps be ignored. The purpose of this paper is to examine various methods of calibrating $p-u$ sound intensity probes, including two near field techniques that might work also in ordinary rooms. 


\section{OUTLINE OF THEORY}

The complex sound intensity can be expressed in terms of the cross spectrum between the sound pressure and the particle velocity, ${ }^{3,6}$

$$
I_{r}+j J_{r}=S_{p u},
$$

where $I_{r}$ is the active intensity, $J_{r}$ is the reactive intensity, and $S_{p u}$ is the cross spectrum. However, the "true" particle velocity is not directly available; therefore the available signal from the particle velocity transducer must be corrected in phase and in amplitude by multiplying with a complex transfer function, $H_{\hat{u} u}$. This function can be determined by exposing the $p-u$ intensity probe to sound field conditions where the specific acoustic impedance is known. The ratio of the "true" specific acoustic admittance in the sound field at the position where the $p-u$ intensity probe is placed during calibration, $H_{p u}$, to the corresponding measured frequency response between the signals from the probe, $H_{p \hat{u}}$, provides the correction of the particle velocity signal relative to the pressure signal, to be used in subsequent measurements of the complex sound intensity as follows: ${ }^{3,6}$

$$
S_{p \hat{u}} H_{\hat{u} u}=S_{p \hat{u}} \frac{H_{p u}}{H_{p \hat{u}}}=S_{p u}=I_{r}+j J_{r},
$$

where $S_{p \hat{u}}$ is the measured cross spectrum between the sound pressure and the particle velocity.

\section{A. Far field calibration in an anechoic room}

The simplest solution would be to expose the device under test to a propagating plane wave in which the specific acoustic admittance equals the reciprocal of the characteristic impedance of the medium,

$$
H_{p u}^{(1)}=\frac{1}{\rho c} .
$$

However, one cannot obtain plane wave conditions at low frequencies even in the largest room, but must correct for the change in phase and amplitude associated with a finite distance to the source. ${ }^{3}$ If the source can be assumed to be a monopole a distance of $r$ from the observation point then Eq. (3) becomes

$$
H_{p u}^{(2)}=\frac{1}{\rho c}\left(1+\frac{1}{j k r}\right),
$$

where $k$ is the wave number. (Note that the $e^{j \omega t}$ convention is used in this paper.) Figure 1, which shows the ratio of $H_{p u}^{(2)}$ to $H_{p u}^{(1)}$, demonstrates that the phase shift associated with the finite distance cannot be neglected below a few hundred hertz even at a distance of $4 \mathrm{~m}$. No ordinary loudspeaker resembles a monopole in its near field, and therefore a distance of several meters is needed. Thus a very special source or a large anechoic room of high quality is required.

\section{B. A monopole on a rigid plane baffle}

If the sound field could be generated by a real monopole one might use Eq. (4) also very near the source, perhaps even without an anechoic room. Unfortunately it is very difficult

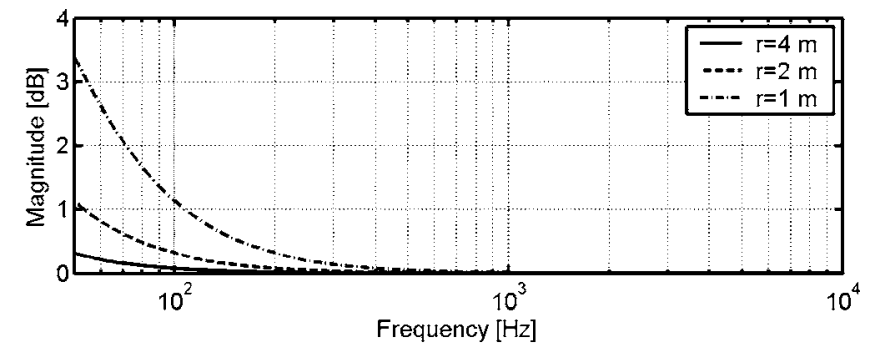

(a)

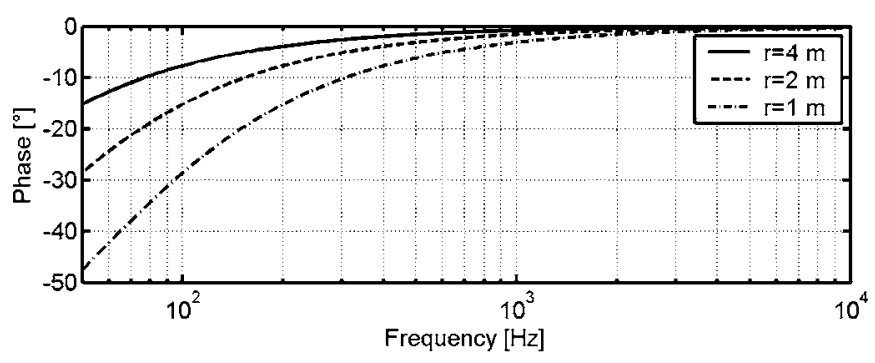

(b)

FIG. 1. Magnitude (a) and phase (b) of the normalized specific acoustic admittance at three different distances in the far field of a monopole.

to construct a "real monopole," that is, an omnidirectional source that can cover a wide frequency range. On the other hand, a small circular hole in a large plane baffle, driven by an enclosed loudspeaker on the other side of the baffle, might approximate a monopole on a baffle and thus generate a simple spherical sound field in the half-space in front of the baffle. In principle the hole should be as small as possible, and the $p-u$ intensity probe should be placed very near the hole. However, in practice the dramatic increase of the particle velocity level relative to the sound pressure level very near a monopole, the need for a well-defined distance between the hole and the transducer, the influence of scattering caused by the transducer, and the influence of reflections from the edges of the baffle call for a compromise. Figure 2 shows the ratio of $H_{p u}^{(2)}$ to $H_{p u}^{(1)}$ in the near field of a monopole on a baffle.

If the hole is small compared with the wavelength then the sound field inside the hole is one-dimensional, and one can improve Eq. (4) by regarding the resulting source as a piston on a baffle. At a distance of $r$ from a baffled, circular piston of radius $b$ the specific acoustic admittance is ${ }^{17}$

$$
\begin{aligned}
H_{p u}^{(3)}= & \frac{1}{2 \rho c}\left(1+\frac{r}{\sqrt{r^{2}+b^{2}}}-j\left(1-\frac{r}{\sqrt{r^{2}+b^{2}}}\right)\right. \\
& \left.\times \cot \left(\frac{k}{2}\left[\sqrt{r^{2}+b^{2}}-r\right]\right)\right)
\end{aligned}
$$

on the axis of the piston. Figure 3 shows the ratio of $H_{p u}^{(3)}$ to $H_{p u}^{(2)}$ at a distance of $5 \mathrm{~cm}$ from the baffle. It can be seen that the influence of a finite radius is fairly small except when the distance $r$ is comparable to the diameter $2 b$. Note that the magnitude of the specific acoustic impedance is reduced by a finite source, in agreement with the fact that the acoustic center of a piston on a baffle is behind the actual surface. ${ }^{18}$ 


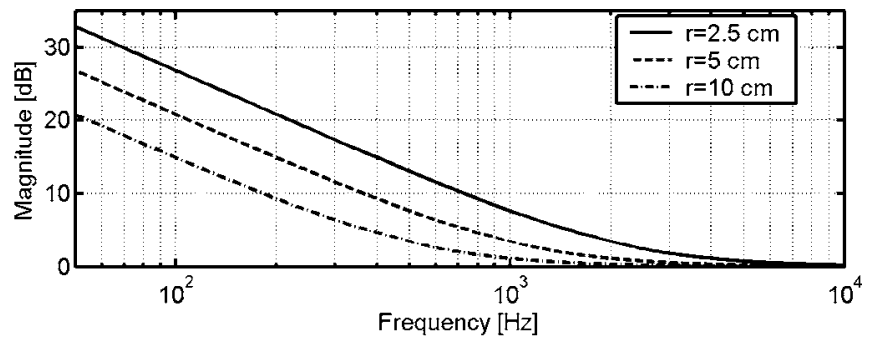

(a)

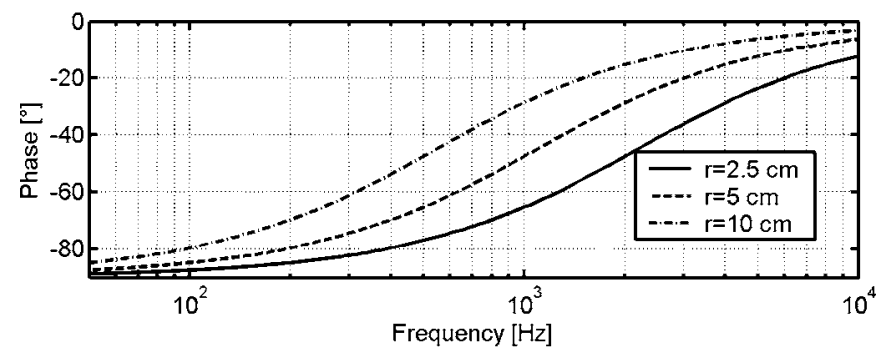

(b)

FIG. 2. Magnitude (a) and phase (b) of the normalized specific acoustic admittance at three different distances in the near field of a monopole on a plane baffle.

\section{A monopole on a rigid spherical baffle}

In practice a plane baffle must obviously be finite, and thus there will inevitably be reflections from the edges. Vibrations caused by the loudspeaker reaction might also be a problem. On the other hand a spherical baffle has no edges and can easily be made very stiff. Thus another solution might be to let the source be a small hole in a hollow rigid sphere driven by a loudspeaker inside the sphere. In the sound field generated by a point source on a rigid sphere the specific acoustic admittance on the axis has the value ${ }^{19}$

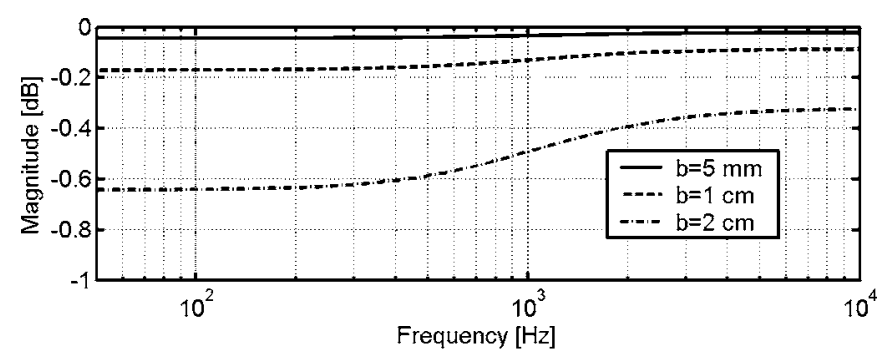

(a)

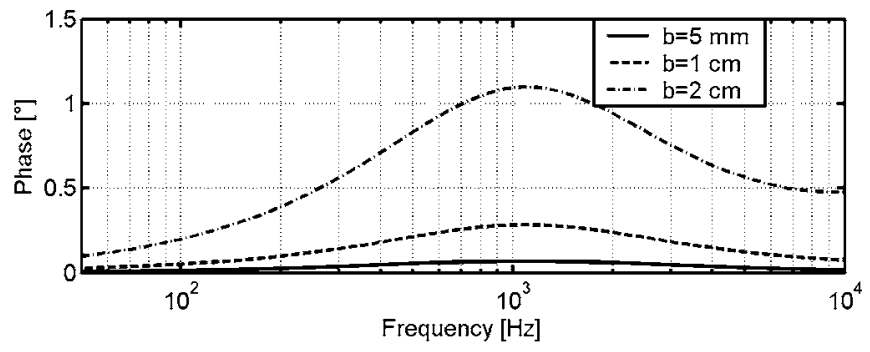

(b)

FIG. 3. The effect of the radius of a circular piston, $b$, on a plane baffle on the (a) magnitude and (b) phase of the specific acoustic admittance at a position $5 \mathrm{~cm}$ from the piston.

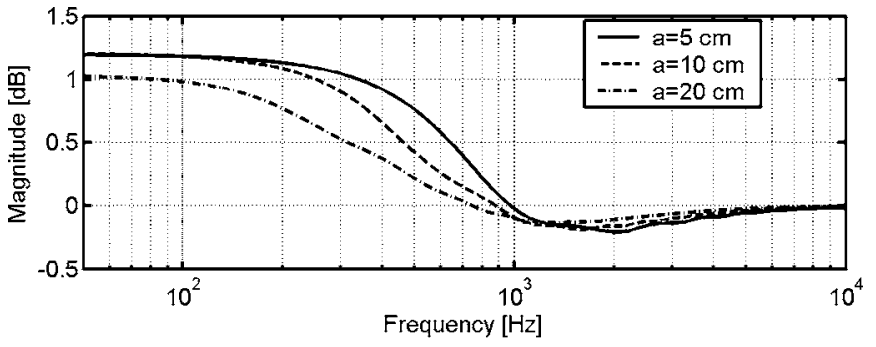

(a)

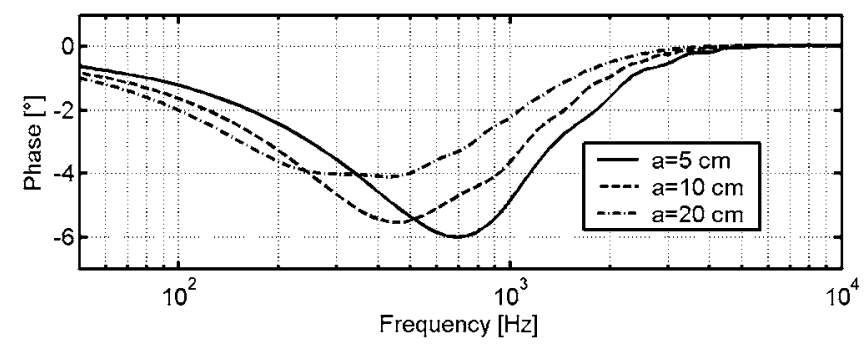

(b)

FIG. 4. Magnitude (a) and phase (b) of the ratio of the specific acoustic admittance $5 \mathrm{~cm}$ from a monopole on a rigid sphere of radius $a$ to the specific acoustic admittance $5 \mathrm{~cm}$ from a monopole on a plane baffle.

$$
H_{p u}^{(4)}=\frac{j}{\rho c} \frac{\sum_{m=0}^{\infty}\left(m+\frac{1}{2}\right) \frac{h_{m}^{\prime}(k r)}{h_{m}^{\prime}(k a)}}{\sum_{m=0}^{\infty}\left(m+\frac{1}{2}\right) \frac{h_{m}(k r)}{h_{m}^{\prime}(k a)}},
$$

where $a$ is the radius of the sphere, $r$ is the distance from the observation point to the center of the sphere, $h_{m}$ is the spherical Hankel function of the second kind and order $m$, and $h_{m}^{\prime}$ is its derivative.

Figure 4 shows the ratio of the specific acoustic impedance in front of a monopole on a spherical baffle, $H_{p u}^{(4)}$, to the specific acoustic admittance in front of a monopole on a planar baffle, $H_{p u}^{(2)}$, at a distance of $5 \mathrm{~cm}$. This ratio is close to unity, indicating that the two admittances are similar. Note that the specific acoustic admittance at a given position in front of a monopole on a sphere at low frequencies is larger than the specific acoustic admittance in front of a monopole on a plane baffle, in agreement with the fact that the acoustic center of a monopole on a sphere is in front of the physical source whereas the acoustic center of a monopole on a plane baffle coincides with the source. ${ }^{18}$ Note also the small irregularities between 1 and $5 \mathrm{kHz}$; they are due to interference between the direct wave and a wave that has traveled around the sphere. In the limit of $a \rightarrow 0$ and $a \rightarrow \infty$ Eq. (6) approaches Eq. (4).

Since the hole in the sphere cannot be infinitely small one might regard it as a small piston of radius $b$ rather than a point source. In this case the specific acoustic admittance becomes ${ }^{19}$ 


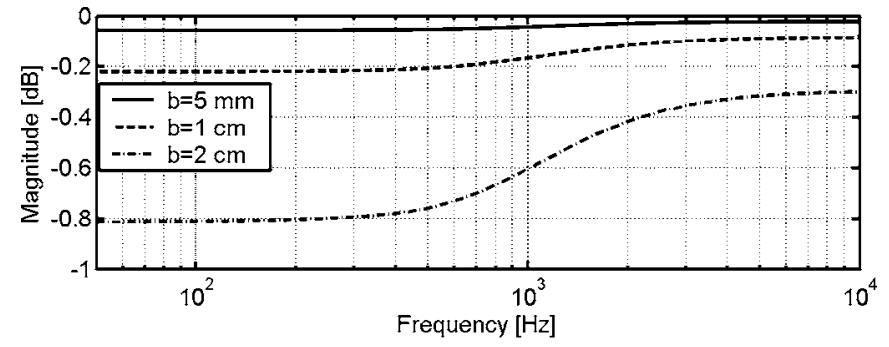

(a)

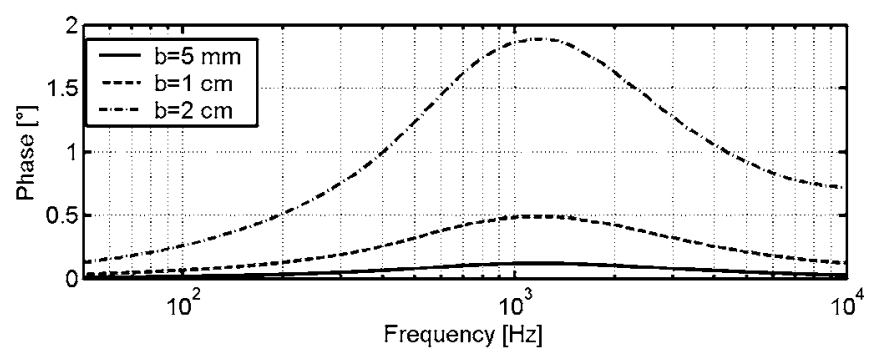

(b)

FIG. 5. The effect of the radius of a circular piston, $b$, on a rigid sphere with a radius of $10 \mathrm{~cm}$ on the magnitude (a) and phase (b) of the specific acoustic admittance at a position $5 \mathrm{~cm}$ from the piston.

$$
H_{p u}^{(5)}=\frac{j}{\rho c} \frac{\sum_{m=0}^{\infty}\left(P_{m-1}(\cos \alpha)-P_{m+1}(\cos \alpha)\right) \frac{h_{m}^{\prime}(k r)}{h_{m}^{\prime}(k a)}}{\sum_{m=0}^{\infty}\left(P_{m-1}(\cos \alpha)-P_{m+1}(\cos \alpha)\right) \frac{h_{m}(k r)}{h_{m}^{\prime}(k a)}},
$$

where $\alpha=\arcsin (b / a)$ and $P_{m}$ is the Legendre function of order $m$.

The ratio of $H_{p u}^{(5)}$ to $H_{p u}^{(4)}$ is shown in Fig. 5. As can be seen the effect of a finite size of the piston on a sphere is similar to but somewhat larger than the effect of a finite piston on a plane baffle.

\section{Two methods based on an impedance tube}

Yet another possibility is to use a standing wave tube with a rigid termination. Under such conditions the specific acoustic admittance is

$$
H_{p u}^{(6)}=\frac{j \tan (k l)}{\rho c},
$$

where $l$ is the distance between the transducer and the rigid termination. Obviously this method breaks down when this distance is a multiple of a quarter of a wavelength. Moreover, viscothermal losses must be taken into account unless the distance is relatively short, ${ }^{20}$ which suggests that $l$ should be less than a quarter of a wavelength at the highest frequency at which the underlying assumption of plane waves holds good. It is not possible to cover the entire frequency range of interest with the tube method.

Alternatively one might measure the frequency response between the particle velocity signal and the sound pressure at the rigid termination using a reference microphone at the termination, and then compensate for the difference between the pressure channel of the $p-u$ probe and the reference mi-

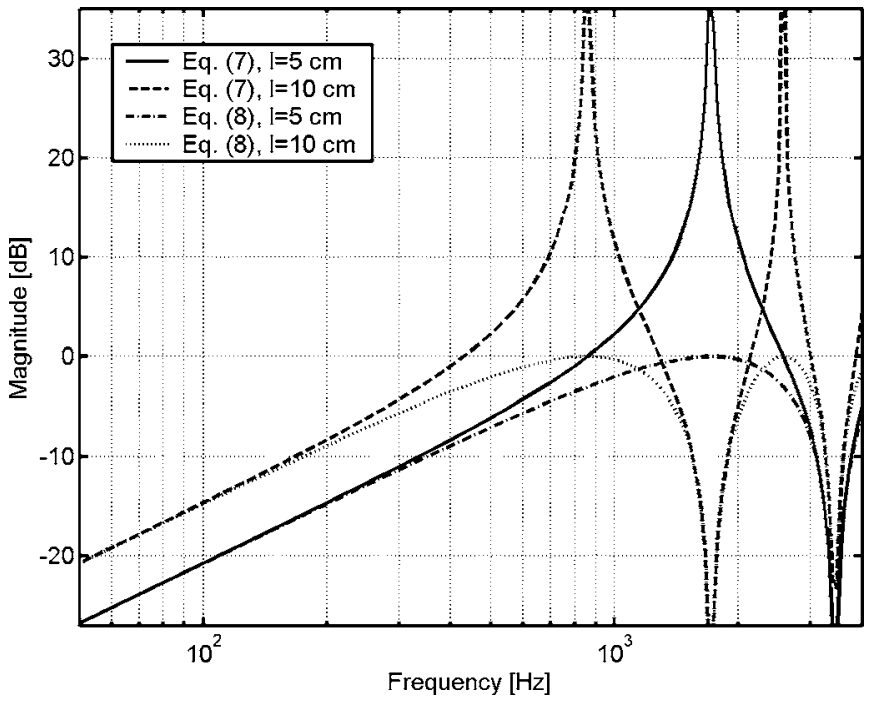

FIG. 6. Magnitude of the normalized specific acoustic admittance at two different positions in a tube with a rigid termination, and magnitude of the normalized ratio of the particle velocity at the same positions to the pressure at the rigid termination.

crophone by placing the $p-u$ probe next to the reference microphone at the termination in a subsequent measurement. The ratio of the particle velocity to the pressure at the termination is

$$
H_{p u}^{(7)}=\frac{j \sin (k l)}{\rho c},
$$

and this expression gives useful results an octave above Eq. (8) as can be seen in Fig. 6. Equations (8) and (9) can easily be extended to take account of viscous and thermal propagation losses in the tube and thermal losses at the rigid termination, ${ }^{20}$ and this has also been tried. However, with the tube dimensions used in the investigation described in what follows the effect of such losses is completely negligible.

\section{EXPERIMENTAL RESULTS}

The two near field methods described in Sec. II have been examined both in a large anechoic room that provides a good approximation to free-field conditions down to $50 \mathrm{~Hz},{ }^{21}$ in an ordinary room of about $180 \mathrm{~m}^{3}$ and a reverberation time of about $0.5 \mathrm{~s}$, and in a small office. In the anechoic room the far field method was also applied since, presumably, this method is the most accurate one. In all cases a Brüel and Kjær (B\&K) "Pulse" analyzer of type 3560 in one-twelfth octave mode was used (although the results presented in what follows are plotted in one-third octave bands). The device under test was a Microflown $\frac{1}{2}$ inch $p-u$ sound intensity probe. Three sources were used in these experiments. In the far field measurements the source was a $60 \mathrm{~mm}$ diameter two-way "coincident-source" loudspeaker unit produced by KEF, mounted in a rigid plastic sphere with a diameter of $270 \mathrm{~mm}$. The "monopole on an infinite baffle" was a wooden IEC baffle for loudspeaker testing with dimensions $1.35 \times 1.65 \mathrm{~m}$ with a $20 \mathrm{~mm}$ diameter hole (with a brass ring so as to reduce flow noise caused by the high air velocity) driven by a conventional small enclosed loudspeaker unit 


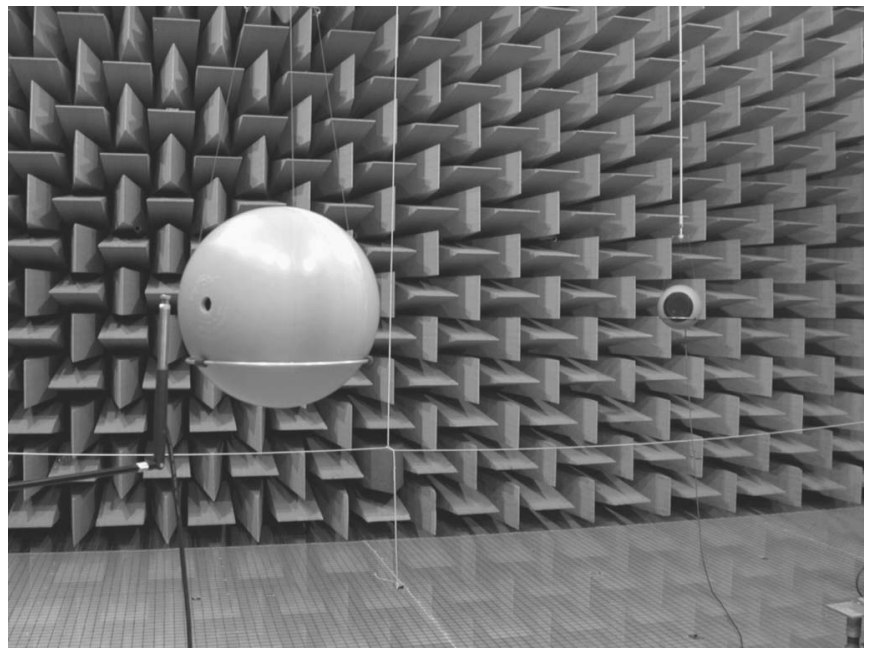

FIG. 7. The Microflown $p-u$ probe close to the experimental "monopole on a sphere" in the anechoic room. In the background the experimental "piston on a sphere" can be seen.

produced by VIFA behind the baffle. The "monopole on a sphere" was $90 \mathrm{~mm}$ VIFA unit mounted inside a rigid plastic sphere with a diameter of $270 \mathrm{~mm}$ with a $20 \mathrm{~mm}$ diameter hole in front of the loudspeaker. Figure 7 shows the Microflown $p-u$ intensity probe close to the "monopole on a sphere" in the anechoic room. In the background the KEF loudspeaker mounted in a sphere can be seen.

All loudspeakers were driven with signals generated by the "Pulse" analyzer and passed through a one-third octave band equalizer (GE27, produced by Rane). Before each measurement the equalizer was adjusted so as to get the flattest possible response of the sound pressure and particle velocity signals. The "frequency-band coherence" between these two signals ${ }^{22}$ turned out to be useful for finding the best setting of the equalizer; the best results were obtained with a frequency-band coherence of unity in the entire frequency range.

Figure 8 shows the amplitude and phase correction of the $p-u$ probe measured with the KEF loudspeaker in the anechoic room at four different distances from $27 \mathrm{~cm}$ to $7.2 \mathrm{~m}$. The measured frequency responses have been processed using Eq. (7), that is, assuming that the source can be modeled as a piston on a sphere. The strange behavior of the phase at 8 and $10 \mathrm{kHz}$ is probably due to the irregular pressure response of the $p-u$ probe in this frequency range. ${ }^{3}$ Close examination reveals some small irregularities in the amplitude and phase determined at the longest distance where the source has probably been too close to the wedges of the anechoic room, but on the whole the results agree within $\pm 0.3 \mathrm{~dB}$ and $\pm 1^{\circ}$ above $100 \mathrm{~Hz}$, as can be seen in Fig. 9, which shows the same data as Fig. 8, but normalized with the correction determined at $70 \mathrm{~cm}$ distance. The data have also been processed using Eq. (4), that is, assuming that the loudspeaker can be modeled as a monopole. The results (not shown) are very similar, but the agreement is slightly better with the piston-on-a-sphere model [Eq. (7)], in particular at the shortest distance. Accordingly, the correction based on this model and data obtained at a distance of $70 \mathrm{~cm}$ are used as a reference in what follows. It should be mentioned that
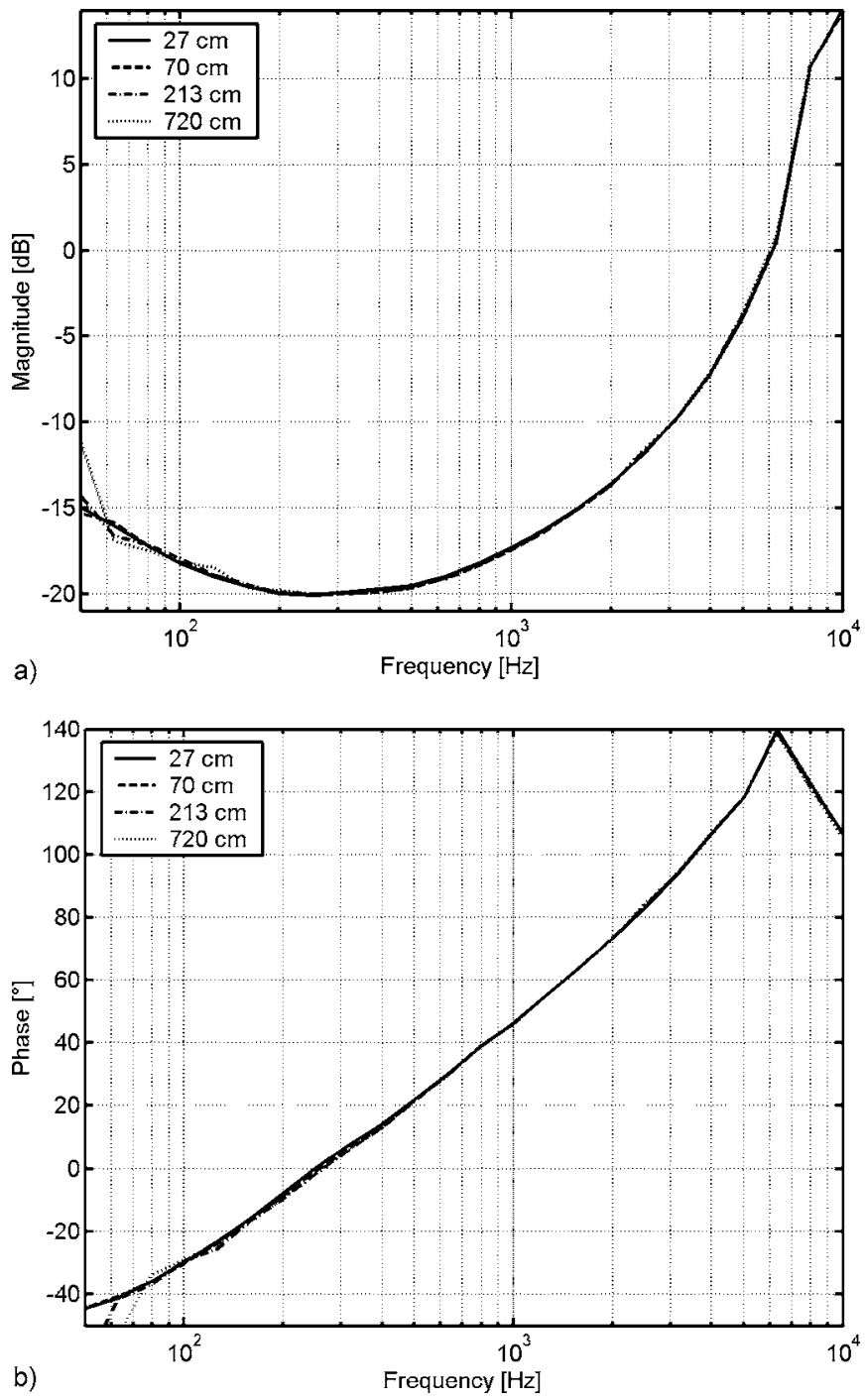

FIG. 8. Amplitude (a) and phase (b) calibration of the Microflown $p$ - $u$ probe determined at four different distances from the KEF loudspeaker mounted in a sphere in the anechoic room.

similar corrections determined in the same anechoic room at different distances from a more conventional two-way loudspeaker (Rogers LS3/5A "Monitor Loudspeaker") did not agree nearly as well at low frequencies, ${ }^{3}$ confirming that a "coincident source" loudspeaker mounted in a sphere approximates a monopole (and, of course, a piston on a sphere) far better than an ordinary loudspeaker in a rectangular box.

Figure 10 shows the amplitude and phase correction determined at three different distances from the "monopole on a sphere" in the anechoic room, normalized with the reference calibration. Although it hardly matters the expression that takes account of the finite size of the hole [Eq. (7)] was used in processing the measured data. Between $63 \mathrm{~Hz}$ and $1.6 \mathrm{kHz}$ the results agree with the reference measurement within $\pm 0.5 \mathrm{~dB}$ and $\pm 2^{\circ}$. The agreement is less perfect but still quite good between 2 and $8 \mathrm{kHz}$.

Figure 11 shows the results of similar measurements at two different distances from the "monopole on a sphere" in the ordinary room. Above $100 \mathrm{~Hz}$ the results are similar to the results obtained with the same source in the anechoic room, although there are more erratic (small) variations with 


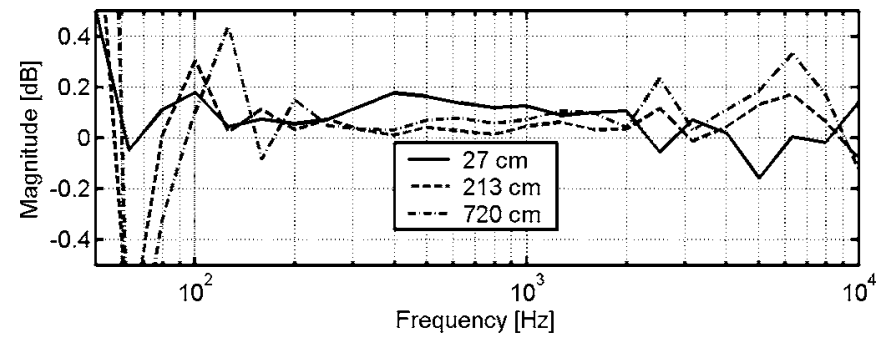

(a)

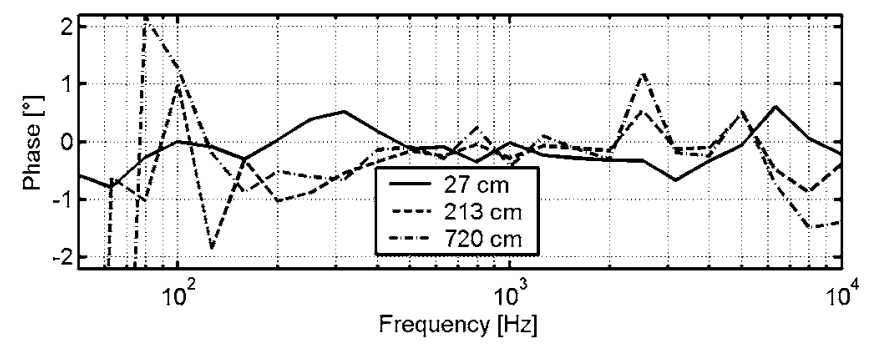

(b)

FIG. 9. Amplitude (a) and phase (b) calibration determined at three different distances from the KEF loudspeaker in the anechoic room relative to the calibration determined at a distance of $70 \mathrm{~cm}$.

the frequency, but for some reason the amplitude seems to have been shifted about $0.7 \mathrm{~dB}$. Results obtained at other positions in the same room and in the small office (not shown) were very similar except below $100 \mathrm{~Hz}$.

Figure 12 shows the results of the measurements close to the "monopole on a plane baffle" in the anechoic room. Also in this case the presumably most accurate expression that takes account of the finite dimension of the hole [Eq. (5)] was used. In this case somewhat larger systematic deviations (within $\pm 4^{\circ}$ ) between the phase and the reference phase occur. These deviations, which could also be seen in similar measurements carried out in the ordinary room (not shown),

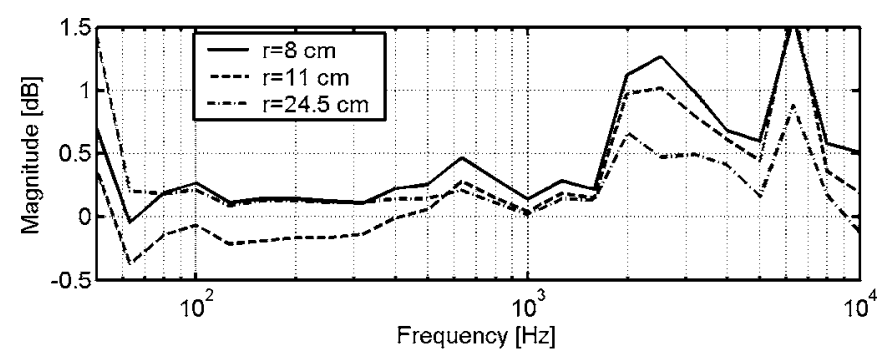

(a)

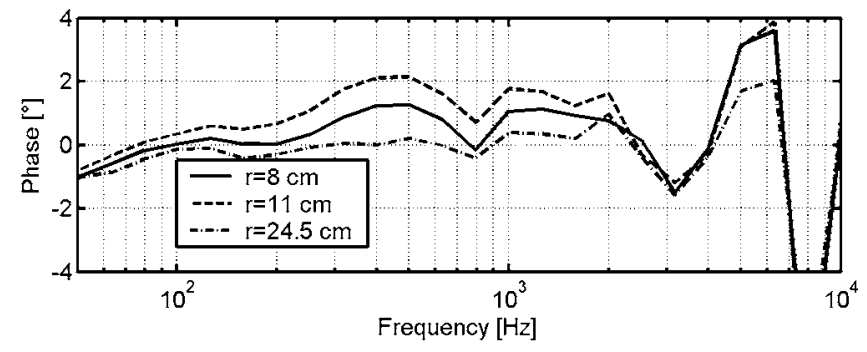

(b)

FIG. 10. Amplitude (a) and phase (b) calibration determined at three different distances from the "monopole on a sphere" in the anechoic room relative to the reference calibration.

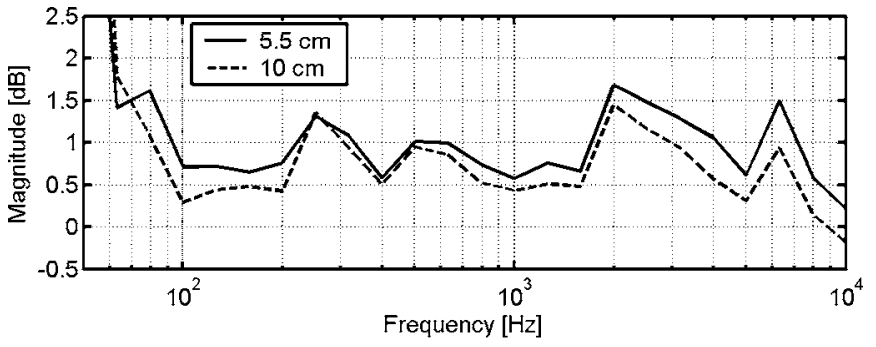

(a)

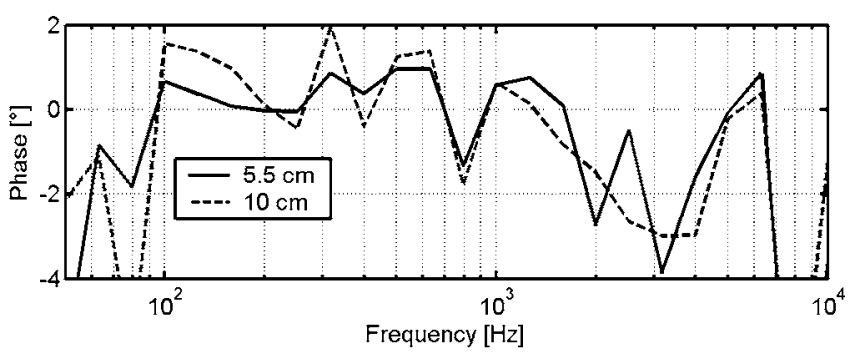

(b)

FIG. 11. Amplitude (a) and phase (b) calibration determined at two different distances from the "monopole on a sphere" in an ordinary room relative to the reference calibration.

are undoubtedly caused by reflections from the edges of the baffle. The same inexplicable tendency to overestimation of the amplitude correction by about $0.7 \mathrm{~dB}$ as observed in Fig. 11 can also be seen here.

Figure 13 shows a tube with a reference microphone (a $\mathrm{B} \& \mathrm{~K}$ microphone of type 4192) at the rigid termination. The tube is driven by a loudspeaker at the other end, and the resonances are damped by absorbing material placed in front of the loudspeaker. The holes for the transducers are tightened with rubber rings, and holes not used are blocked by solid brass plugs. The distance $l$ is $5 \mathrm{~cm}$, so in principle the

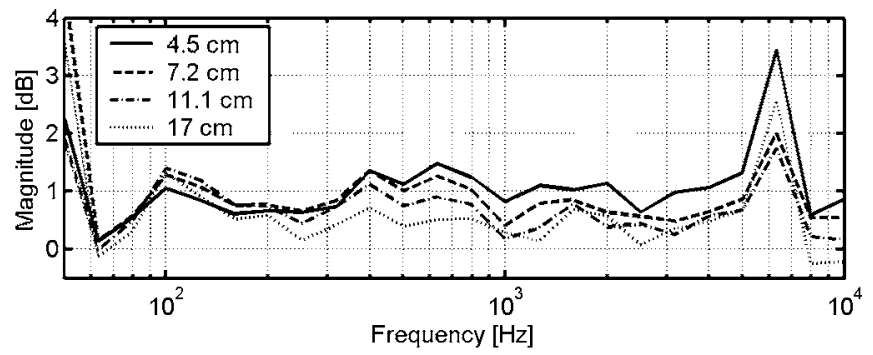

(a)

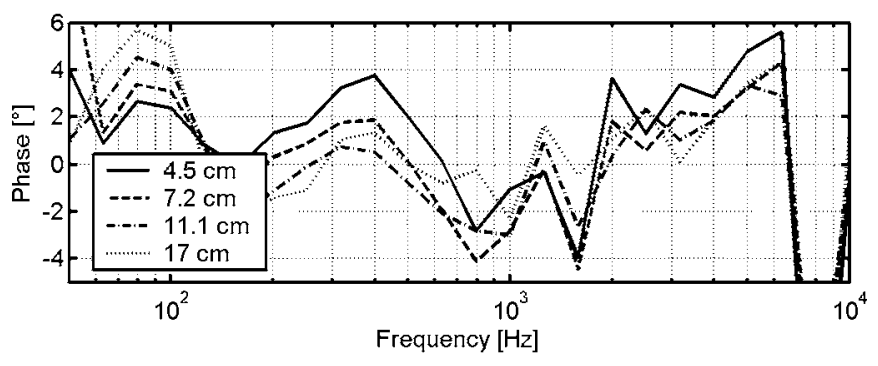

(b)

FIG. 12. Amplitude (a) and phase (b) calibration determined at four different distances from the "monopole on a plane baffle" in the anechoic room relative to the reference calibration. 


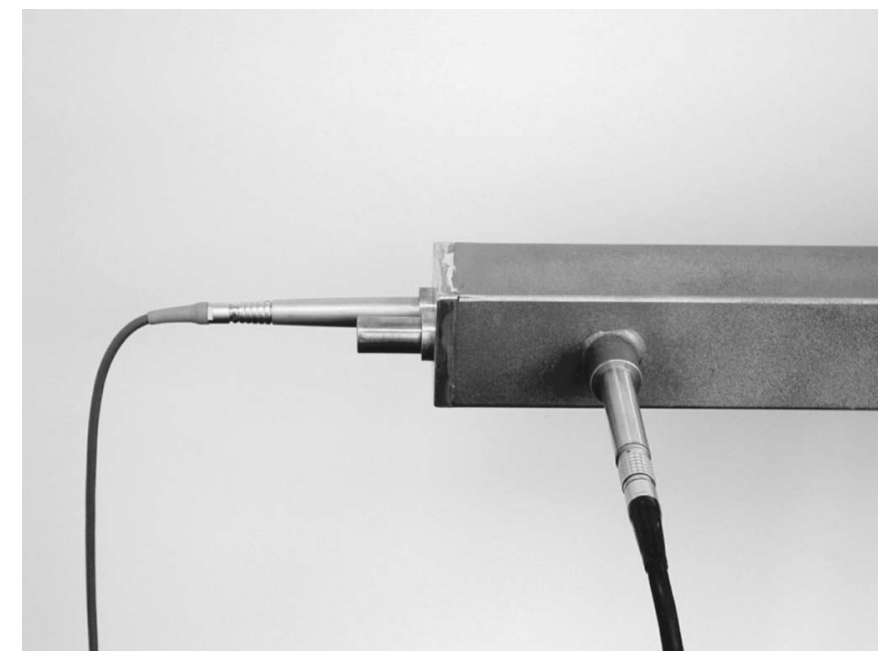

FIG. 13. Tube with the Microflown $p-u$ probe mounted $5 \mathrm{~cm}$ from the rigid termination and $\mathrm{B} \& \mathrm{~K}$ microphone mounted at the termination.

device should work up to $1.7 \mathrm{kHz}$ if the "direct" method based on Eq. (8) is used and up to $3.4 \mathrm{kHz}$ if the alternative method involving a reference microphone is used (cf. Fig. 6). In this case the "Pulse" analyzer was used in the FFT mode with a spectral resolution of $1 \mathrm{~Hz}$. Figure 14 shows a comparison of the resulting amplitude and phase corrections with the reference measurement from the anechoic room. The two amplitude corrections agree with the reference within $1 \mathrm{~dB}$, and the two phase corrections agree with the reference within $4^{\circ}$ except above $1.5 \mathrm{kHz}$. There is no obvious explanation for the systematic underestimation of the amplitude seen between 200 and $400 \mathrm{~Hz}$ and the systematic overestimation of the phase beween 300 and $600 \mathrm{~Hz}$.

\section{DISCUSSION}

It seems clear that the most accurate calibration method requires a large anechoic room. However, from a practical point of view the near field method based on a "monopole on a sphere" is more interesting. The most obvious contribution to the measurement uncertainty with this method is associated with determining the physical distance $r$ in Eq. (6) [or Eq. (7)]. If the uncertainty on a "true" distance of $5 \mathrm{~cm}$ amounts to, say, $1 \mathrm{~mm}$, then the resulting uncertainty will take values up to $0.2 \mathrm{~dB}$ and $0.6^{\circ}$. On the other hand, increasing the distance inevitably increases the influence of deviations from perfect free-field conditions. Reflections of extraneous noise from the sphere may disturb the weak pressure signal (cf. Fig. 2), and this problem is probably most serious if the transducer is very close to the sphere. A distance of $5 \mathrm{~cm}$ seems a good compromise. However, it is clear from the experimental results that there are other contributions to the resulting uncertainty than the uncertainty on the distance.

The small but apparently systematic deviations seen in Fig. 10(a) between 2 and $6.3 \mathrm{kHz}$, the small but systematic overestimation seen in Figs. 11(a) and 12(a), and the small deviations seen in Figs. 14(a) and 14(b) may perhaps be due to the transducer under test rather than the calibration procedures. It is apparent from Figs. 1, 2, and 6 that the specific
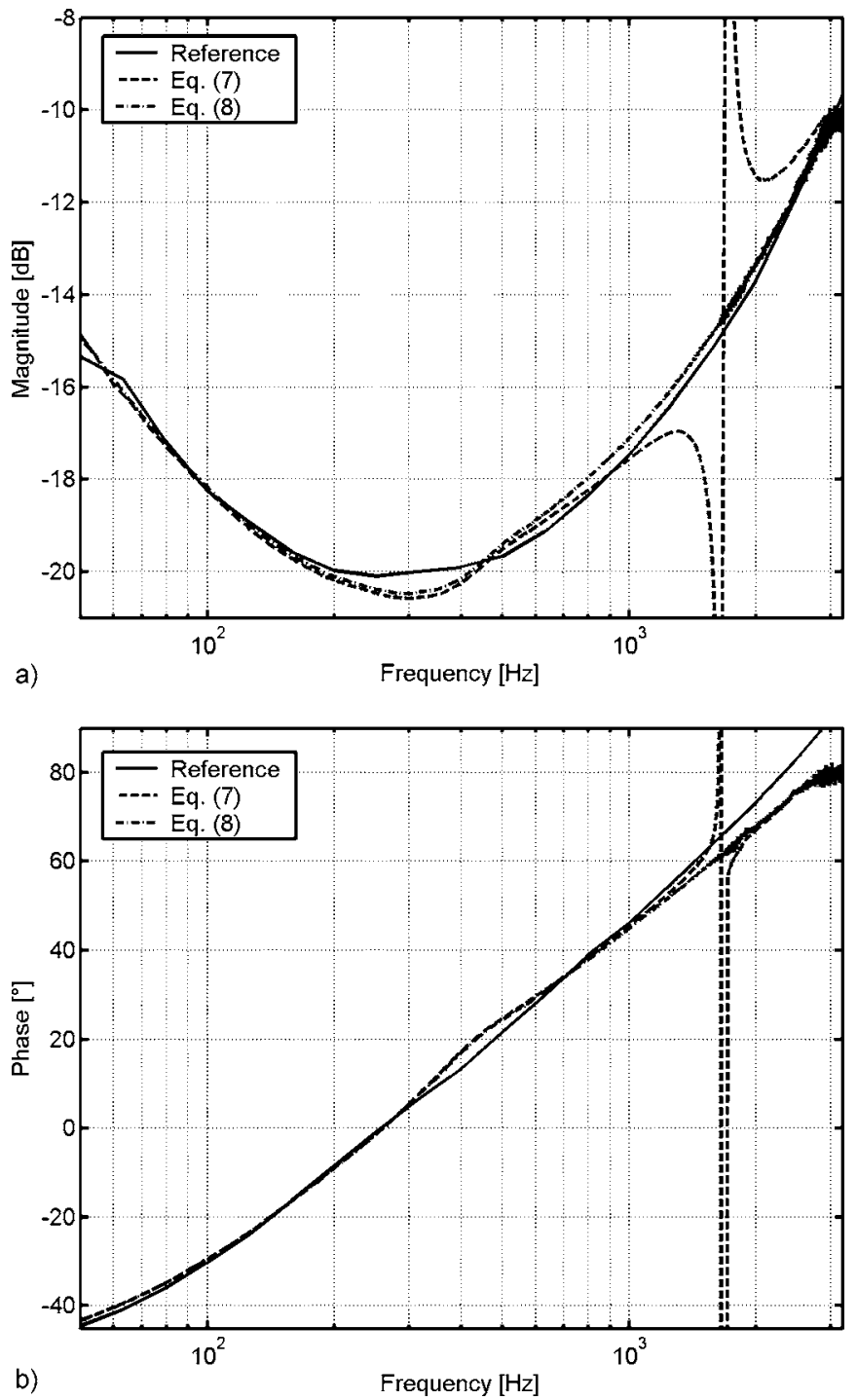

FIG. 14. Amplitude (a) and phase (b) calibration determined in the tube using the methods based on Eq. (8) and Eq. (9). The reference calibration determined in the anechoic room is also shown.

acoustic admittance takes values that vary over an interval of $40 \mathrm{~dB}$, and perhaps the particle velocity transducer is not perfectly linear.

The required accuracy of the calibration depends, of course, on the application of the $p-u$ intensity probe. In Ref. 8 it was concluded that reliable measurement of absorption coefficients with such a device calls for calibration errors within $0.5 \mathrm{~dB}$ and $2^{\circ}$ unless the sample under test is highly absorbing, in which case somewhat larger errors can be tolerated. The results presented in the foregoing indicate that this accuracy can be achieved in a large anechoic room of good quality. However, it seems that it is only just possible to satisfy this requirement with the "monopole on a sphere" in an ordinary room.

The analysis presented in Ref. 3 showed that the required accuracy of the phase calibration of a $p-u$ intensity probe used in sound power measurements depends on whether the measurements take place in the reactive near field of the source under test or not. If near fields are avoided then fairly large phase errors, say $\pm 10^{\circ}$, can be tolerated. 
However, if the measurement surface is close to a source the ratio of the reactive to the active intensity may well take values of up to $10 \mathrm{~dB}$ at low frequencies, ${ }^{3,23}$ and then even a phase error of $\pm 2.5^{\circ}$ is unacceptable (the resulting error in the estimated sound power would be $1.6 \mathrm{~dB} /-2.5 \mathrm{~dB}$ ). With extremely reactive sound field conditions, as in the experiments with the loudspeaker dipole described in Ref. 3, only a very good calibration carried out in a large anechoic room will be good enough. If an anechoic room of adequate quality is not available then the only way of obtaining a phase correction of satisfactory accuracy seems to be to use the phase adjustment technique described in Ref. 3 .

The investigation presented here has concentrated on calibrating the particle velocity transducer relative to the pressure microphone of the $p-u$ intensity probe. However, it is perhaps worth mentioning that absolute calibration of a particle velocity transducer may be possible with the "monopole on a sphere" using the sound pressure behind the loudspeaker inside the sphere as a reference. This pressure, which can be measured with a calibrated condenser microphone, is proportional to the volume displacement.

\section{CONCLUSIONS}

A number of methods of calibrating a $p-u$ sound intensity probe have been examined. The most accurate method requires an anechoic room of good quality and a "coincident source" loudspeaker mounted in a sphere. If the anechoic room is sufficiently large then an ordinary loudspeaker placed far from the transducer under test can be used instead. A near field method involving sound emitted from a hole in a hollow rigid sphere gives slightly less accurate results, but has the significant advantage that it can be used in the field. Alternatively, a similar near field method with a plane baffle can be used, also in the field, but this method is less accurate than the method based on a sphere since reflections from the edges of the baffle cannot be avoided. Finally, it is possible to calibrate in an impedance tube, although only in a limited frequency range.

\section{ACKNOWLEDGMENTS}

The authors would like to thank Microflown for lending us a $p-u$ intensity probe. The authors would also like to thank Vicente Cutanda for the idea of using a spherical baffle.

${ }^{1}$ H.-E. de Bree, "The Microflown: An acoustic particle velocity sensor," Acoust. Aust. 31, 91-94 (2003).
${ }^{2}$ R. Raangs, W. F. Druyvesteyn, and H.-E. de Bree, "A low-cost intensity probe," J. Audio Eng. Soc. 51, 344-357 (2003).

${ }^{3}$ F. Jacobsen and H. E. de Bree, "A comparison of two different sound intensity measurement principles," J. Acoust. Soc. Am. 118, 1510-1517 (2005).

${ }^{4}$ IEC Publication 61043, Instruments for the Measurement of Sound Intensity—Measurements with Pairs of Pressure Sensing Microphones (International Electrotechnical Commission, Geneva, Switzerland, 1993).

${ }^{5}$ American National Standard ANSI S1.9-1996, Instruments for the Measurement of Sound Intensity (American National Standards Institute, New York, 1996).

${ }^{6}$ F. J. Fahy, Sound Intensity, 2nd ed. (E \& FN Spon, London, 1995).

${ }^{7}$ H.-E. de Bree, R. Lanoye, S. de Cock, and J. van Heck, In situ, broad band method to determine the normal and oblique reflection coefficient of acoustic materials, SAE technical paper 2005-01-2443, SAE noise and vibration conference and exhibition, Grand Traverse, MI, USA, 2005.

${ }^{8}$ Y. Liu and F. Jacobsen, "Measurement of absorption with a $p-u$ sound intensity probe in an impedance tube," J. Acoust. Soc. Am. 118, 21172120 (2005).

${ }^{9}$ F. Jacobsen and Y. Liu, "Near field acoustic holography with particle velocity transducers," J. Acoust. Soc. Am. 118, 3139-3144 (2005).

${ }^{10} \mathrm{R}$. Visser, "Acoustic source localization based on pressure and particle velocity measurements," Proceedings of Inter-Noise 2003, Jeju, Korea, 2003, pp. 665-670.

${ }^{11} \mathrm{R}$. Visser, A boundary element approach to acoustic radiation and source identification, Ph.D. thesis, University of Twente, The Netherlands, 2004.

${ }^{12} \mathrm{O}$. Wolff and R. Sottek, Panel contribution analysis-An alternative window method, SAE technical paper 2005-01-2273, SAE noise and vibration conference and exhibition, Grand Traverse, MI, USA, 2005.

${ }^{13}$ H.-E. de Bree and W. F. Druyvesteyn, A particle velocity sensor to measure the sound from a structure in the presence of background noise, Proceedings of forum acusticum 2005, Budapest, Hungary, 2005.

${ }^{14}$ T. B. Gabrielson, D. L. Gardner, and S. L. Garret, "A simple neutrally buoyant sensor for direct measurement of particle velocity and intensity in water," J. Acoust. Soc. Am. 97, 2227-2237 (1995).

${ }^{15}$ K. Kim, T. B. Gabrielson, and G. C. Lauchle, "Development of an accelerometer-based underwater acoustic intensity sensor," J. Acoust. Soc. Am. 116, 3384-3392 (2004).

${ }^{16}$ K. J. Bastyr, G. Lauchle, and J. A. McConnel, "Development of a velocity gradient underwater acoustic intensity sensor," J. Acoust. Soc. Am. 106, 3178-3188 (1999).

${ }^{17} \mathrm{~K}$. Beissner, "On the plane-wave approximation of acoustic intensity," J. Acoust. Soc. Am. 71, 1406-1411 (1982).

${ }^{18}$ F. Jacobsen, S. Barrera Figueroa, and K. Rasmussen, "A note on the concept of acoustic center," J. Acoust. Soc. Am. 115, 1468-1473 (2004).

${ }^{19}$ E. G. Williams, Fourier Acoustics. Sound Radiation and Nearfield Acoustical Holography (Academic Press, London, 1999).

${ }^{20}$ S. W. Rienstra and A. Hirschberg, An introduction to acoustics, Technische Universiteit Eindhoven, 1999.

${ }^{21}$ F. Ingerslev, O. J. Pedersen, P. K. Møller, and J. Kristensen, "New rooms for acoustic measurements at the Danish Technical University," Acustica 19, 185-199 (1968).

${ }^{22} \mathrm{~F}$. Jacobsen, "Active and reactive, coherent and incoherent sound fields," J. Sound Vib. 130, 493-507 (1989).

${ }^{23} \mathrm{~F}$. Jacobsen and H.-E. de Bree, Intensity-based sound power determination under adverse sound field conditions: $p$ - $p$ probes versus $p-u$ probes, Proceedings of Twelfth International Congress on acoustics and vibration, Lisbon, Portugal, 2005. 